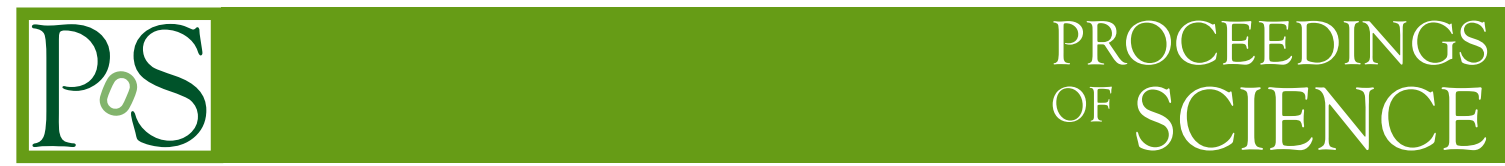

\title{
Smoking Gun for GRB in Milky Way?
}

\author{
Janusz Ziółkowski* \\ Copernicus Astronomical Center, \\ ul. Bartycka 18, 00-716 Warsaw, Poland \\ E-mail: jzdcamk.edu.p]
}

I will start this review with considering the youngest neutron star and the youngest black hole in our Galaxy. Then, I will describe the supernova remnant W49B that probably harbors the youngest galactic black hole. The properties of this remnant suggest that it originated about one thousand years ago during a bipolar/jet driven type Ib/Ic supernova explosion. This event could be associated with a gamma ray burst in our Galaxy.

Frontier Research in Astrophysics,

26-31 May 2014

Mondello (Palermo), Italy

${ }^{*}$ Speaker. 


\section{Introduction}

The search for young very compact stellar remnants such as neutron stars or black holes is not very difficult because they are in most cases surrounded by an expanding nebula of supernova remnant (SNR) that could be observed for several tens of thousands of years. In the case of neutron stars it is additionally easier because the compact object itself is usually seen as a source of a characteristic thermal radiation from its still hot surface. Additionally, it is frequently seen as a radio and/or a X-ray pulsar or a gamma-ray pulsar. In this way, we are reasonably sure that the youngest neutron star seen in our Galaxy is the one in the SNR Cas A. Its age is only about 330 years.

In the case of young black holes the situation is not so easy because we can observe only an expanding SNR. It is not difficult to estimate its age but the presence of a black hole in its interior can be established only by circumstantial arguments. Such task was recently successfully carried out by Lopez et al. (2013) who found the convincing arguments indicating that a thousand years old SNR W49B most likely contains a black hole. At this moment this unseen object is the youngest known black hole in our Galaxy.

\section{Neutron star in SNR Cas A}

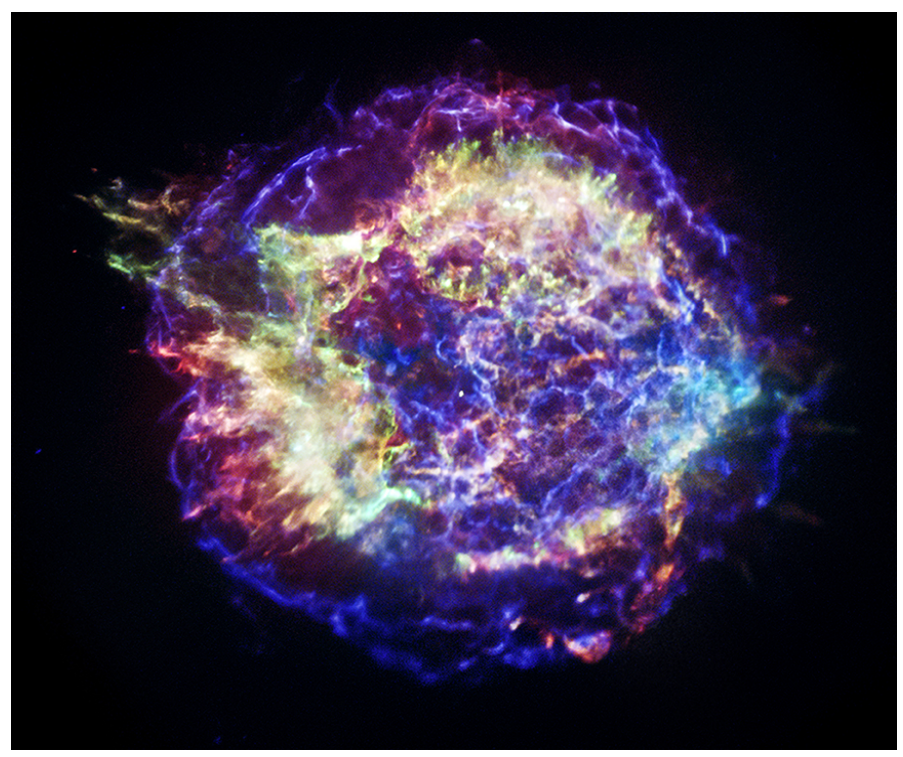

Figure 1: X-ray image of Cas A taken by Chandra (from http://chandra.harvard.edu). The small white dot in the center of the picture is the young neutron star.

Cas A is a very young SNR located at a distance of $3.4 \mathrm{kpc}$. Its age estimated from the nebula expansion rate is only $\sim 330$ years (Fesen et al. 2006). The supernova that exploded around 1680 was not observed optically except possibly one proposed but controversial sighting by Flamsteed in August 1680 (Stephenson \& Green 2003). The explosion left a neutron star discovered by Chandra in 1999 (Tananbaum 1999). The neutron star is located at the center of the nebula (Fig. 1) and its 
X-ray emission shows a thermal spectrum consistent with the emission from the surface of a young neutron star with the present temperature of $2 \times 10^{6} \mathrm{~K}$. In spite of many efforts no evidence for pulsations in X-ray emission was found (Halpern \& Gotthelf 2010). No radio or optical counterpart was detected (McLaughlin et al. 2001, Fesen et al. 2006). However, there are little doubts that the source of the emission is a neutron star because Chandra observations detected rapid cooling of the surface temperature of the source, consistent with the cooling of a young neutron star with carbon atmosphere. Heinke \& Ho (2010) found a $3.6 \pm 0.6 \%$ decrease in surface temperature (21 $\%$ flux decrease) over 9 yr period (Fig. 2). Elshamouty et al. (2013) using larger body of Chandra observations found temperature decrease of $2.9 \pm 0.5_{\text {stat }} \pm 1.0_{\text {sys }} \%$ over $10 \mathrm{yr}$ period. In any case, there are no doubts that we are seeing a very young rapidly cooling neutron star.

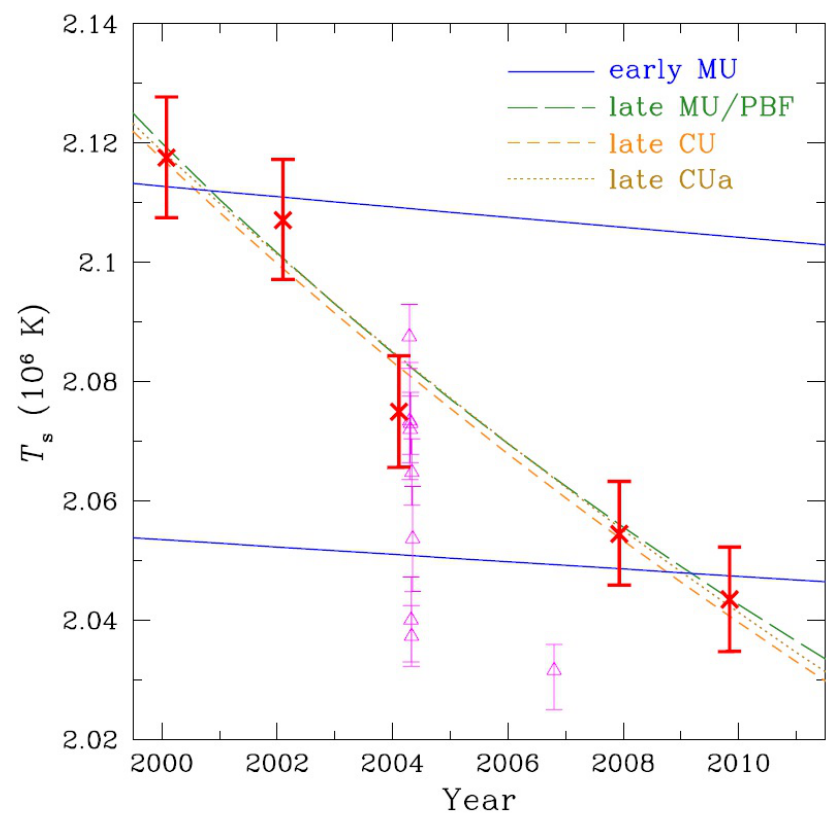

Figure 2: Surface temperature $T_{\mathrm{S}}$ of the Cas A neutron star, obtained from spectral fits of Chandra observations, as a function of time (crosses and triangles indicate best-fit values with $1 \sigma$ errorbars). Curves are fits (to well-calibrated data, the 5 red crosses) with a simple model for neutron star cooling by different mechanisms; see Heinke \& Ho (2010) for explanation. Figure after Heinke \& Ho (2010).

\section{Unusual SNR W49B}

W49B is a young galactic SNR with a number of highly interesting and unique properties. It is located at a distance of about $10 \mathrm{kpc}$ (Hwang et al. 2000) and its age is estimated to be about 1000 year (Hwang et al. 2000, Immler \& Kuntz 2005). W49B is one of the most luminous Galactic SNR in X-rays (with $L_{\mathrm{X}} \sim 10^{38} \mathrm{erg} / \mathrm{s}$ (Immler \& Kuntz 2005)). It is also very bright in $\gamma$-rays, as observed by Fermi LAT (Abdo et al. 2010).

The X-ray spectrum of W49B has strong emission lines from ions of several metals with super solar abundances, indicative of their ejecta-dominated origin (Lopez et al. 2013). 
The morphology of W49B is quite unique. W49B is the only Galactic SNR with large-scale segregation of its nucleosynthetic products (Lopez et al. 2009, 2011). This is particularly true about iron. Generally, iron shows higly supersolar abundances, but its distribution is highly asymmetric: it is missing from the Western half of the SNR. This segregation indicates highly asymmetric SN explosion. Such bipolar explosions were investigated by Umeda \& Nomoto (2008). They found that bipolar explosions have much greater nickel yields (comparing with the symmetric explosions). These yields increase with asphericity, progenitor mass and explosion energy.

The physical origin of W49B's unusual properties could be due to a bipolar/jet-driven explosion of a massive star (Lopez et al. 2013). A bipolar/jet-driven Type Ib/Ic SN origin is consistent with the median abundance ratios from a spatially- resolved spectroscopic analysis using XMMNewton observations (Lopez et al. 2009), and it may account for the anisotropic distribution of iron since heavy elements are preferentially ejected along the polar axis of the progenitor in these explosions (Khokhlov et al. 1999, Mazzali et al. 2001, Ramirez-Ruiz \& MacFadyen 2010, Couch et al. 2011). Also more recent observations by Chandra (Lopez et al. 2013) support the conclusion about a bipolar/jet-driven explosion leading to the formation of W49B. In addition to forming untypical SNR such explosion is expected to form a black hole rather than a neutron star.

Based on radio observations, the rate of such events is estimated to be $0.7_{-0.6}^{+1.6} \%$ of local Type $\mathrm{Ib} / \mathrm{Ic} \mathrm{SNe}$ (Soderberg et al. 2010), equating to 1 per $10^{3}-10^{4}$ years per galaxy (Lopez et al. 2011). Subset of these .explosions might be hypernovae or GRBs (Izzard et al. 2004; Podsiadlowski et al. 2004).

\section{Nature of the compact object in SNR W49B}

Theoretical models predict that a bipolar/jet-driven explosion leads to the formation of black hole as a compact remnant. There are also other arguments supporting the presence of a black hole in W49B. From 220-ks Chandra observations Lopez et al. (2013) estimated that any undetected neutron stars would have an upper limit X-ray luminosity of $L_{X} \leq 3.7 \times 10^{31} \mathrm{erg} / \mathrm{s}$ in the $0.1-10 \mathrm{keV}$ band. Comparing this limit with the predicted luminosity of a 1000 year old neutron star (see Fig. 4) they find that the observational limit is 2-3 orders of magnitude lower than predictions of neutron star cooling models. Also extensive search for radio pulsations in W49B using Arecibo (Gorham et al. 1996) gave negative results. Finally, the spectrum of the bright gamma-ray emission detected with Fermi-LAT from W49B is inconsistent with it arising from a pulsar (Abdo et al. 2010). It is therefore reasonable to assume that the compact object produced in the explosion which created W49B is a black hole.

\section{GRB connection?}

It is, of course, tempting to ask whether a bipolar/jet-driven explosion leading to the formation of a black hole was not associated with a GRB in our Galaxy. Such possibility was indicated in the Chandra press release of June 2, 2004 (http://chandra.harvard.edu/press/04_releases/press_060204.html). This communicate motivated Ioka et al. (2004) to model the high-energy photon emission around GRB remnants caused by possible ultra high energy cosmic rays (UHECRs) from the GRBs (Waxman, 1995, 2004). They applied the results to W49B and found that if GRBs are indeed sources 


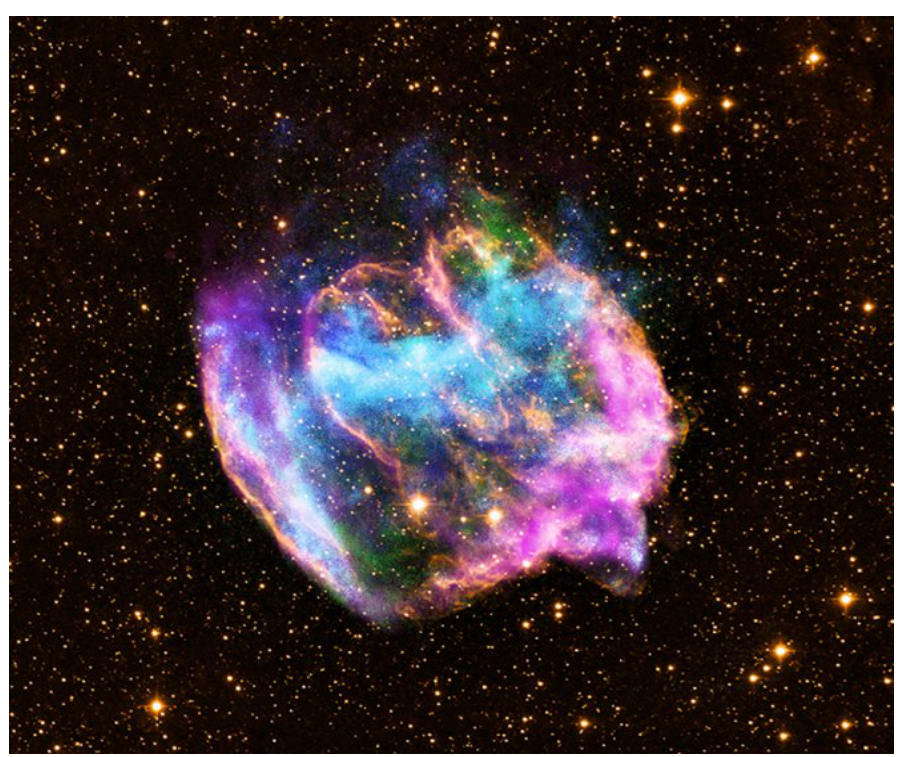

Figure 3: X-ray image of W49B taken by Chandra (from http://chandra.harvard.edu).

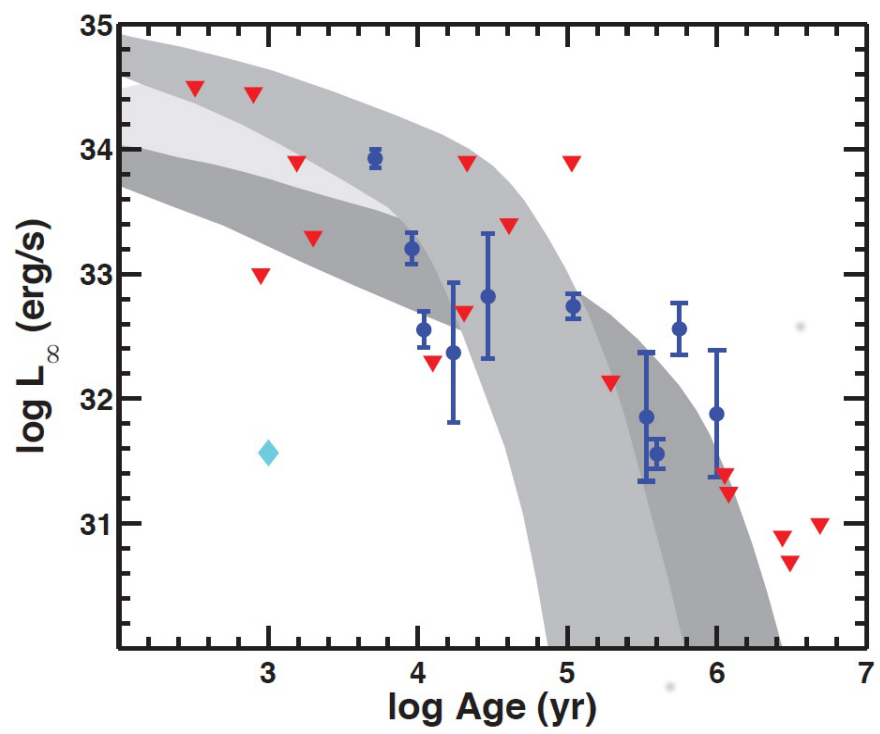

Figure 4: Plot of total photon luminosity at infinity $L_{\infty}$ versus age for nearby neutron stars and predictions from models of neutron star cooling. The light blue diamond is Lopez et al. (2013) upper limit for a hypothetical neutron star in W49B; dark blue points are detections and red triangles are upper limits, from Tsuruta et al. (2009). Shaded bands are model predictions of Page et al. (2006) for neutron star cooling with a heavy element envelope (dark gray), a maximum amount of light elements (medium gray), and an intermediate amount of light elements (light gray). Lopez et al. (2013) upper limit for W49B $\left(\log L_{\infty} \leq 31\right.$.6) is two to three orders of magnitude below the predictions for a 1000 year old source. Figure after Lopez et al. (2013). 
of UHECRs, a natural consequence of identification of W49B as a GRB remnant would be expectation of a detectable $\mathrm{TeV}$ photon emission around W49B. This region was observed by H.E.S.S. telescope and indeed the emission in the $0.3-10 \mathrm{TeV}$ band was detected with a statistical significance of $8.8 \sigma$ (Brun et al., 2011). The future C.T.A. observations will produce substantially more precise imaging of the emission surrounding $\mathrm{W} 49 \mathrm{~B}$ and might provide new important constraints on the jet structure of the GRBs.

\section{Conclusion}

The morphological, spectral, and environmental characteristics of W49B are indicating that it originated in a bipolar/jet-driven Type Ib/Ic SN explosion. There are strong arguments that W49B contains a youngest known galactic black hole. It is possible that the origin of W49B was associated with a GRB in our Galaxy. If it was indeed the case, then the future C.T.A. observations of the $\mathrm{TeV}$ emission surrounding W49B (detected by H.E.S.S.) might provide important constraints on the jet structure of the GRBs.

\section{Acknowledgments}

I would like to thank the anonimous referee for calling my attention to the H.E.S.S. observation of W49B. This work was partially supported by the Polish National Science Center project 2012/04/M/ST9/00780.

\section{References}

[1] A.A. Abdo, M. Ackermann, M. Ajello, et al. Fermi-LAT Study of Gamma-ray Emission in the Direction of Supernova Remnant W49B, ApJ 722 (2010) 1303 [arXiv:1008.4190]

[2] F. Brun, M. de Naurois, W. Hofmann, S. Carrigan, A. Djannati-Ataï \& S. Ohm The W49 region as seen by H.E.S.S., PoS 201 (2011) 545

[3] S.M. Couch, D. Pooley, J.C. Wheeler \& M. Milosavljevic Aspherical Supernova Shock Breakout and the Observations of Supernova $2008 D$ ApJ 727 (2011) 104 [arXiv:1007.3693]

[4] K.G. Elshamouty, C.O. Heinke, G.R. Sivakoff et al. Measuring the Cooling of the Neutron Star in Cassiopeia A with all Chandra X-Ray Observatory Detectors, ApJ 777 (2013) 22 [arXiv:1306.3387]

[5] R.A. Fesen, M.C. Hammell, J. Morse et al. The Expansion Asymmetry and Age of the Cassiopeia A Supernova Remnant, ApJ 645 (2006) 283 [arXiv:astro-ph/0603371]

[6] P.W. Gorham, P.S. Ray, S.B. Anderson et al. A Pulsar Survey of 18 Supernova Remnants, ApJ 458 (1996) 257

[7] J.P. Halpern \& E.V. Gotthelf Spin-Down Measurement of PSR J1852+0040 in Kesteven 79: Central Compact Objects as Anti-Magnetars, ApJ 709 (2010) 436 [arXiv:0911.0093]

[8] C.O. Heinke \& W.C.G. Ho Direct Observation of the Cooling of the Cassiopeia A Neutron Star, ApJ 719 (2010) L167 [arXiv:1007.4719] 
[9] U. Hwang, R. Petre \& J.P. Hughes The X-Ray Line Emission from the Supernova Remnant W49B, ApJ 532 (2000) 970 [arXiv:astro-ph/9911323]

[10] S. Immler \& K.D. Kuntz Discovery of X-Ray Emission from Supernova 1970G with Chandra: Filling the Void between Supernovae and Supernova Remnants, ApJ 632 (2005) L99 [arXiv:astro-ph/0506023]

[11] K. Ioka, Shiho Kobayashi \& P. Meszaros Extended GeV-TeV Emission around Gamma-Ray Burst Remnants and the Case of W49B, ApJ 613 (2004) L17 [arXiv:astro-ph/0406555]

[12] R.G. Izzard, E. Ramirez-Ruiz \& C.A. Tout Formation rates of core-collapse supernovae and gamma-ray bursts, MNRAS 3 (2004) 1215 [arXiv:astro-ph/0311463]

[13] A.M. Khokhlov, P.A. Hoflich, E.S. Oran et al. Jet-induced Explosions of Core Collapse Supernovae, ApJ 524 (1999) L107 [arXiv:astro-ph/9904419]

[14] L.A. Lopez, E. Ramirez-Ruiz, D. Castro \& S. Pearson The Galactic Supernova Remnant W49B Likely Originates from a Jet-driven, Core-collapse Explosion, ApJ 764 (2013) L50 [arXiv:1301.0618]

[15] L.A. Lopez, E. Ramirez-Ruiz, D. Huppenkothen et al. Using the X-ray Morphology of Young Supernova Remnants to Constrain Explosion Type, Ejecta Distribution, and Chemical Mixing, ApJ 732 (2011) 114 [arXiv:1011.0731]

[16] L.A. Lopez, E. Ramirez-Ruiz, D.A. Pooley \& T.E. Jeltema Tools for Dissecting Supernova Remnants Observed with Chandra: Methods and Application to the Galactic Remnant W49B, ApJ 691 (2009) 875 [arXiv:0810.0009]

[17] P.A. Mazzali, K.S. Kawabata, K. Maeda et al. An Asymmetric Energetic Type Ic Supernova Viewed Off-Axis, and a Link to Gamma Ray Bursts, Science 308 (2005) 1284 [arXiv:astro-ph/0505199]

[18] M.A. McLaughlin, J.M. Cordes, A.A. Deshpande et al. Upper Limits on Periodic, Pulsed Radio Emission from the X-Ray Point Source in Cassiopeia A, ApJ 547 (2001) L41 [arXiv:astro-ph/0010338]

[19] D. Page, U. Geppert \& F. Weber The cooling of compact stars, Nuclear Physics A 777 (2006) 497 [arXiv:astro-ph/0508056]

[20] P. Podsiadlowski, P.A. Mazzali, K. Nomoto et al. The Rates of Hypernovae and Gamma-Ray Bursts: Implications for Their Progenitors, ApJ 607 (2004) L17 [arXiv:astro-ph/0403399]

[21] E. Ramirez-Ruiz \& A.I. MacFadyen The Hydrodynamics of Gamma-ray Burst Remnants, ApJ 716 (2010) 1028 [arXiv:0808.3448]

[22] A.M. Soderberg, S. Chakraborti, G. Pignata et al. A relativistic type Ibc supernova without a detected gamma-ray burst, Nature 463 (2010) 513 [arXiv:0908.2817]

[23] F.R. Stephenson \& D.A. Green Book Review: Historical supernovae and their remnants / Oxford University Press, 2002, Astronomy 31 (2003) 118903

[24] H. Tananbaum Cassiopeia A, IAU Circ. 7246 (1999)

[25] S. Tsuruta, J. Sadino, A. Kobelski, et al. Thermal Evolution of Hyperon-Mixed Neutron Stars, ApJ 691 (2009) 621

[26] H. Umeda \& K. Nomoto How Much ${ }^{56 N}$ i Can Be Produced in Core-Collapse Supernovae? Evolution and Explosions of 30-100 M $M_{\text {solar }}$ Stars, ApJ 547 (2001) L41 [arXiv:0707.2598]

[27] Waxman, E. 1995, Phys. Rev. Lett. 75, 386.

[28] Waxman, E. 2004, ApJ 606, 988. 